\title{
Features of Bulk Viscosity Simulation in Carbon Dioxide
}

\author{
E.V. Kustova, M.A. Mekhonoshina \\ Saint-Petersburg State University, St. Petersburg, 199034 Russia \\ mekhonoshinama@gmail.com
}

\begin{abstract}
The aim of this work is to build an adequate model for calculating the bulk viscosity in carbon dioxide and to evaluate its contribution to the normal mean stress under various conditions. Bulk viscosity characterizes the finite time of energy exchange between the translational and internal degrees of freedom

Bulk viscosity in carbon dioxide is considered in the one-temperature approximation developed using the Chapman-Enskog method taking into account the rotational and vibrational degrees of freedom, as well as complex mechanisms of vibrational relaxation including intraand inter-mode vibrational energy transitions. Chemical reactions are not included to the model.

Two models for the bulk viscosity coefficient in $\mathrm{CO}_{2}$ are considered: the model based on the exact kinetic theory methods, as well as the model representing the bulk viscosity coefficient as a sum of independent contributions of rotational and vibrational degrees of freedom. The latter model predicts that in carbon dioxide at room temperature, the bulk viscosity coefficient may exceed that of shear viscosity by several orders of magnitude and, thus, make a significant contribution to the stress tensor. In this work, it is shown that the use of a consistent theoretical approach does not allow separating the bulk viscosity into independent rotational and vibrational contributions. Vibrational relaxation times are evaluated using different models.
\end{abstract}

Keywords: kinetic theory, bulk viscosity, carbon dioxide.

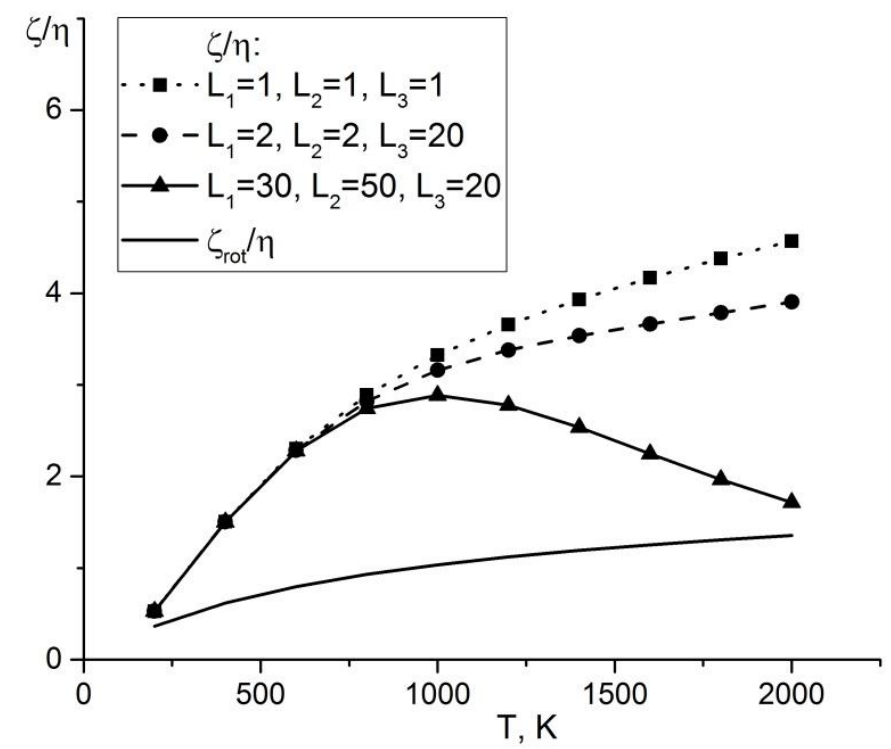

Ratio of bulk and shear viscosity coefficients in $\mathrm{CO}_{2}$ 
УДК 533.6.011

\title{
Особенности моделирования объемной вязкости в углекислом газе
}

\author{
Е.В. Кустова, М.А. Мехоношина \\ Санкт-Петербургский государственный университет, \\ Россия, Санкт-Петербург, 199034, Университетская набережная, 7-9 \\ mekhonoshinama@gmail.com
}

\begin{abstract}
Аннотация
Целью настоящей работы является построение адекватной модели расчета объемной вязкости в углекислом газе и оценка ее вклада в нормальное напряжение при различных условиях. Объемная вязкость характеризует конечное время передачи энергии между поступательным движением частиц и внутренними степенями свободы.

В работе в однотемпературном приближении с помощью метода Чепмена-Энскога рассматривается объемная вязкость углекислого газа с учетом вращательных и колебательных степеней свободы, а также сложных механизмов колебательной релаксации, включая внутри- и межмодовые переходы колебательной энергии. Химические реакции в модели не учитываются.

Рассматриваются две модели для коэффициента объемной вязкости в $\mathrm{CO}_{2}$ : модель, основанная на методах точной кинетической теории, а также модель, представляющая коэффициент объемной вязкости в виде суммы независимых вкладов вращательных и колебательных степеней свободы. Последняя модель предсказывает, что в углекислом газе при комнатной температуре объемная вязкость может на несколько порядков превышать сдвиговую вязкость и, таким образом, вносить значительный вклад в тензор напряжений. В данной работе показано, что применение последовательного теоретического подхода не позволяет разделить объемную вязкость на независимые вклады вращательных и колебательных степеней свободы. Также проведен расчет времени колебательной релаксации на основании различных моделей.
\end{abstract}

Ключевые слова: кинетическая теория, объемная вязкость, углекислый газ.

\section{1. Введение}

Настоящая работа посвящена исследованию объемной вязкости в углекислом газе. Объемная вязкость возникает вследствие конечного времени передачи энергии между поступательным движением частиц и внутренними степенями свободы. Ряд научных коллективов занимается экспериментальными [1-4] и теоретическими исследованиями объемной вязкости [5-13]. Особый интерес представляет изучение объемной вязкости в $\mathrm{CO}_{2}$, так как его сложная молекулярная структура и множественные механизмы релаксации внутренней энергии [14-16] могут существенно влиять на транспортные свойства.

До недавнего времени в вычислительной гидродинамике объемная вязкость полагалась пренебрежимо малой. Несколько лет назад в работе [17] было показано, что при комнатной температуре объемная вязкость в углекислом газе может быть в сотни и тысячи раз больше, чем сдвиговая вязкость и, как следствие, вносить значительный вклад в тензор напряжений. Это предположение было использовано в [18] для изучения структуры ударной волны, были найдены некоторые интересные особенности распределения гидродинамических переменных. С другой стороны, в $[19,20]$ эти результаты не были подтверждены. Таким образом, на данный момент не существует единого мнения по поводу поведения объемной вязкости в $\mathrm{CO}_{2}$. 
Целью настоящей работы является получение достоверной информации о коэффициенте объемной вязкости в углекислом газе при различных условиях методами кинетической теории. Объемная вязкость рассчитывается с учетом связанных колебательных мод $\mathrm{CO}_{2}$, а также сложных механизмов колебательной релаксации, включая внутри- и межмодовые колебательные энергетические переходы. Химические реакции не включены в кинетическую схему, так как нас интересуют свойства однокомпонентного газа. Для получения теоретической модели применяется однотемпературное приближение модифицированного метода Чепмена-Энскога; для получения предварительных количественных результатов используются некоторые упрощения. Предложен метод расчета времени колебательной релаксации. Результаты сравниваются с данными, полученными в [17]; оценивается вклад вращательных и колебательных степеней свободы в объемную вязкость.

\section{2. Модель кинетики}

Однотемпературное приближение кинетической теории строится для случая слабого отклонения от полного равновесия, когда существует следующая связь между характерными временами процессов [9]:

$$
\tau_{t r}<\tau_{r o t}<\tau_{v i b r} \ll \theta
$$

Здесь $\tau_{t r}, \tau_{r o t}, \tau_{v i b r}$ являются характерными временами релаксации поступательных, вращательных и колебательных степеней свободы соответственно; $\theta$ - время изменения макроскопических параметров газа.

При однотемпературном описании течения в отсутствии химических реакций система уравнений гидродинамики не содержит релаксационных уравнений и включает уравнения сохранения массы, импульса и энергии

$$
\begin{gathered}
\frac{\mathrm{d} \rho}{\mathrm{d} t}+\rho \nabla \cdot \mathbf{v}=0, \\
\rho \frac{\mathrm{d} \mathbf{v}}{\mathrm{d} t}+\nabla \cdot \mathbf{P}=0, \\
\rho \frac{\mathrm{d} u}{\mathrm{~d} t}+\nabla \cdot \mathbf{q}+\mathbf{P}: \nabla \mathbf{v}=0
\end{gathered}
$$

Здесь $\rho$ - плотность; $\mathbf{v}$ - скорость газа; $u$ - внутренняя энергия единицы массы; $\mathbf{P}$ - тензор напряжений; $\mathbf{q}$ - тепловой поток. Влияние обменов энергией между поступательными и внутренними степенями свободы в такой постановке проявляется в появлении дополнительного коэффициента теплопроводности внутренней энергии в потоке тепла и коэффициента объемной вязкости в диагональных членах тензора напряжений (см. следующий раздел). Кроме того, при расчете удельной внутренней энергии $u$ необходимо учитывать вращательную и колебательную энергию молекул.

$\mathrm{CO}_{2}$ - линейная трехатомная молекула [21], которая в основном электронном состоянии имеет три колебательные моды: симметричную, деформационную и антисимметричную. Наличие трех колебательных мод вызывает различные обмены колебательной энергией внутри мод и между ними. Кроме того, ангармоничность колебаний приводит к тому, что моды не являются независимыми. Обозначим через $i_{1}, i_{2}, i_{3}$ колебательные квантовые числа, соответствующие симметричной, деформационной и антисимметричной модам. Введем также максимальные числа уровней $\mathrm{L}_{1}, \mathrm{~L}_{2}, \mathrm{~L}_{3}$ в каждой моде, учитываемые при расчетах. Максимально возможные значения, соответствующие энергии диссоциации: $\mathrm{L}_{1}=31$, $\mathrm{L}_{2}=63, \mathrm{~L}_{3}=20$ (при зафиксированных нижних состояниях остальных мод). Кроме того, 
для оценки влияния числа возбужденных состояний на объемную вязкость мы будем задавать другие комбинации $\mathrm{L}_{1}, \mathrm{~L}_{2}, \mathrm{~L}_{3}$.

Быстрыми неупругими процессами, приводящими к релаксации внутренней энергии и, следовательно, ответственными за появление объемной вязкости, являются вращательные и колебательные энергетические обмены. Анализ различных механизмов колебательной релаксации, выполненный в [20], показывает, что доминирующими являются следующие каналы:

$\mathrm{VT}_{2}$ - обмен колебательной и поступательной энергиями при столкновении с инертным партером

$$
\mathrm{CO}_{2}\left(\mathrm{i}_{1}, \mathrm{i}_{2}, \mathrm{i}_{3}\right)+\mathrm{CO}_{2} \rightleftharpoons \mathrm{CO}_{2}\left(\mathrm{i}_{1}, \mathrm{i}_{2} \pm 1, \mathrm{i}_{3}\right)+\mathrm{CO}_{2}
$$

Межмодовые $\mathrm{VV}_{m-k}$ обмены колебательными квантами внутри одной молекулы

$$
\begin{array}{ll}
\mathrm{VV}_{1-2}: & \mathrm{CO}_{2}\left(\mathrm{i}_{1}, \mathrm{i}_{2}, \mathrm{i}_{3}\right)+\mathrm{CO}_{2} \rightleftharpoons \mathrm{CO}_{2}\left(\mathrm{i}_{1} \pm 1, \mathrm{i}_{2} \mp 2, \mathrm{i}_{3}\right)+\mathrm{CO}_{2}, \\
\mathrm{VV}_{2-3}: & \mathrm{CO}_{2}\left(\mathrm{i}_{1}, \mathrm{i}_{2}, \mathrm{i}_{3}\right)+\mathrm{CO}_{2} \rightleftharpoons \mathrm{CO}_{2}\left(\mathrm{i}_{1}, \mathrm{i}_{2} \pm 3, \mathrm{i}_{3} \mp 1\right)+\mathrm{CO}_{2}, \\
\mathrm{VV}_{1-2-3}: & \mathrm{CO}_{2}\left(\mathrm{i}_{1}, \mathrm{i}_{2}, \mathrm{i}_{3}\right)+\mathrm{CO}_{2} \rightleftharpoons \mathrm{CO}_{2}\left(\mathrm{i}_{1} \pm 1, \mathrm{i}_{2} \pm 1, \mathrm{i}_{3} \mp 1\right)+\mathrm{CO}_{2}
\end{array}
$$

Данные VV-обмены (особенно между симметричной и деформационной модами) близки к резонансным, поэтому их вероятность высока. Тем не менее, малый дефект резонанса при VV-обменах должен приводить к тому, что их вклад в коэффициент объемной вязкости будет малым; этот факт проверен в дальнейших расчетах.

\section{3. Вычисление объемной вязкости}

При условии (1) тензор напряжений $\mathbf{P}$ в первом приближении метода Чепмена-Энскога выражается как [9]

$$
\mathbf{P}=p \mathbf{I}-2 \eta \mathbf{S}-\zeta \nabla \cdot \mathbf{v I},
$$

где $p$ - давление; $\mathbf{S}$ - бездивергентный тензор скоростей деформации; $\mathbf{I}-$ единичный тензор; $\eta, \zeta-$ коэффициенты сдвиговой и объемной вязкости.

Для расчета коэффициентов переноса мы используем приближение первого порядка модифицированного метода Чепмена - Энскога; алгоритм вычисления подробно обсуждается в [9].

Коэффициенты сдвиговой $\eta$ и объемной вязкости $\zeta$ для однокомпонентного газа в однотемпературном подходе получены в виде [9]

$$
\begin{gathered}
\eta=\frac{5 k T}{8 \Omega^{(2,2)}}, \\
\zeta=\frac{k T}{\beta_{\text {int }}}\left(\frac{c_{\text {int }}}{c_{V}}\right)^{2}
\end{gathered}
$$

Здесь $\Omega^{(2,2)}$ - интеграл столкновений, рассчитанный с использованием данных [22]; $k$ постоянная Больцмана; $T$ - температура газа; $\beta_{\mathrm{int}}-$ интеграл столкновений, зависящий от дефекта резонанса внутренней энергии при быстрых неупругих столкновениях; $c_{V}=c_{t r}+c_{r o t}+c_{v i b r}=c_{t r}+c_{\text {int }}-$ полная удельная теплоемкость при постоянном объеме; $c_{t r}, c_{r o t}$, $c_{v i b r}$ - компоненты теплоемкости, соответствующие поступательной, вращательной и колебательной энергии. 
Интегральная скобка $\beta_{\text {int }}$ характеризует усредненное изменение внутренней энергии в результате наиболее частых неупругих столкновений [9]

$$
\beta_{\mathrm{int}}=2\left\langle\left(\Delta \boldsymbol{E}^{\mathrm{int}}\right)^{2}\right\rangle
$$

Здесь безразмерное изменение внутренней энергии дается формулой

$$
\Delta \mathcal{E}^{\mathrm{int}}=\frac{\varepsilon_{i_{1}^{\prime} i^{\prime}{ }_{2} i_{3}}+\varepsilon_{j^{\prime}}+\varepsilon_{k_{1}^{\prime} k_{2} k_{2}^{\prime}{ }_{3}}+\varepsilon_{l^{\prime}}-\varepsilon_{i_{1} i_{2} i_{3}}-\varepsilon_{j}-\varepsilon_{k_{1} k_{2} k_{3}}-\varepsilon_{l}}{k T}=\frac{\varepsilon_{i^{\prime} l^{\prime}}+\varepsilon_{k^{\prime} l^{\prime}}-\varepsilon_{i j}-\varepsilon_{k l}}{k T}=\Delta \mathcal{E}^{\text {rot }}+\Delta \mathcal{E}^{v i b r},
$$

где $\varepsilon_{j}$ - вращательная энергия молекулы на $j$-ом уровне, штрихованные индексы соответствуют энергиям после столкновения. В обозначении внутренней энергии $\varepsilon_{i j}$ для удобства вместо квантовых чисел $\left(\mathrm{i}_{1}, \mathrm{i}_{2}, \mathrm{i}_{3}\right)$ используется $i$ для определения колебательного состояния. В данной работе для вращательной энергии используется модель жесткого ротатора; это позволяет разбить изменение энергии на два члена, соответствующие вращательной и колебательной энергии.

Оператор осреднения вводится на основе функции распределения нулевого приближения, которая в однотемпературном подходе представляет собой максвеллбольцмановское распределение по скорости и по внутренней энергии [9]

$$
\left\langle\left(\Delta \varepsilon^{\mathrm{int}}\right)^{2}\right\rangle=\left(\frac{k T}{2 \pi m}\right)^{1 / 2} \sum_{i j k l i^{\prime} j^{\prime} k^{\prime} l^{\prime}} \frac{s_{i j} s_{k l}}{Z_{\text {int }}^{2}} \int\left(\Delta \varepsilon^{\mathrm{int}}\right)^{2} \gamma^{3} \exp \left(-\gamma^{2}-\frac{\varepsilon_{i j}}{k T}-\frac{\varepsilon_{k l}}{k T}\right) \sigma_{i j k l}^{i^{\prime} j^{\prime} k^{\prime} l^{\prime} \mathrm{d}^{2} \Omega \mathrm{d} \gamma}
$$

Здесь $s_{i j}$ - внутренний статистический вес; $Z_{\text {int }}-$ внутренняя статистическая сумма; для жестких ротаторов $Z_{\text {int }}=Z_{r o t} Z_{v i b r} ; \gamma$ - безразмерная относительная скорость; $\sigma_{i j k l}^{i^{\prime} j^{\prime} k ' l^{\prime}}-$ дифференциальное сечение неупругого столкновения, приводящего к изменению внутренней энергии; $\mathrm{d}^{2} \Omega$ - телесный угол. На основе уравнения (6) можно ввести время релаксации внутренней энергии $[5,9,23]$

$$
\frac{1}{\tau_{\text {int }}}=\frac{k n}{m c_{\text {int }}} \beta_{\text {int }},
$$

тогда выражение для объемной вязкости примет следующий вид:

$$
\zeta=\frac{k^{2} T n}{m}\left(\frac{c_{\text {int }}}{c_{V}}\right)^{2} \frac{\tau_{\text {int }}}{c_{\text {int }}}
$$

Обычно в экспериментах вместо полного времени релаксации внутренней энергии измеряются времена релаксации вращательной $\tau_{\text {rot }}$ и колебательной $\tau_{v i b r}$ энергии. Поэтому полезно выразить коэффициент объемной вязкости в терминах $\tau_{r o t}$ и $\tau_{v i b r}$. Для этой цели воспользуемся предположением Мэзона и Мончика [23], что столкновения с одновременным обменом вращательной и колебательной энергией редки, поэтому сечения столкновений для колебательных энергетических переходов не зависят от вращательных состояний и наоборот. Это позволяет расщепить интеграл в формуле (5) на два члена, связанных с вращательной $\left(\Delta \mathcal{E}^{r o t}\right)^{2}$ и колебательной $\left(\Delta \mathcal{E}^{v i b r}\right)^{2}$ энергией. Заметим, что в приведенном выше предположении интеграл от произведения $\Delta \mathcal{E}^{r o t}$ и $\Delta \mathcal{E}^{v i b r}$ равен нулю. Тогда для интегральных скобок будет верно, что $\beta_{\text {int }}=\beta_{r o t}+\beta_{v i b r}$, а время релаксации внутренней энергии можно записать в виде 


$$
\frac{c_{\text {int }}}{\tau_{\text {int }}}=\frac{c_{r o t}}{\tau_{r o t}}+\frac{c_{v i b r}}{\tau_{v i b r}}
$$

Наконец, коэффициент объемной вязкости можно представить в следующем виде:

$$
\zeta=\frac{k^{2} T n}{m}\left(\frac{c_{\text {int }}}{c_{V}}\right)^{2}\left(\frac{c_{r o t}}{\tau_{r o t}}+\frac{c_{v i b r}}{\tau_{v i b r}}\right)^{-1}
$$

Стоит отметить, что в известной работе Крамера [17] коэффициент объемной вязкости разбивается на два независимых слагаемых

$$
\zeta=\zeta^{r o t}+\zeta^{v i b r}
$$

и каждый коэффициент выражается через соответствующее время релаксации

$$
\zeta^{r o t}=\frac{k^{2} T n}{m}\left(\frac{c_{r o t}}{c_{V}}\right)^{2} \frac{\tau_{r o t}}{c_{r o t}}, \quad \zeta^{v i b r}=\frac{k^{2} T n}{m}\left(\frac{c_{v i b r}}{c_{V}}\right)^{2} \frac{\tau_{v i b r}}{c_{v i b r}}
$$

В следующем разделе мы сравниваем коэффициенты объемной вязкости, рассчитанные по этим моделям.

\section{4. Результаты}

Обсудим сначала времена релаксации вращательной и колебательной энергии. Время вращательной релаксации вычисляется по модели Паркера [24] при условии, что $\tau_{r o t}$ не зависит от колебательного спектра молекулы. Расчет времени колебательной релаксации в $\mathrm{CO}_{2}$ является более сложной задачей из-за множества релаксационных каналов. Имеющиеся экспериментальные данные существуют только для некоторых релаксационных процессов [25]. Для расчета общего времени колебательной релаксации мы предлагаем следующий алгоритм. Определим $\tau_{v i b r}$ как результат всех столкновений, ведущих к изменению колебательной энергии

$$
\frac{1}{\tau_{v i b r}}=\frac{2 k n}{m c_{v i b r}}\left\langle\left(\Delta \boldsymbol{E}^{v i b r}\right)^{2}\right\rangle
$$

Предполагая, что сечения колебательных энергетических переходов не зависят от вращательных состояний, после некоторых преобразований получаем

$$
\left\langle\left(\Delta \boldsymbol{E}^{v i b r}\right)^{2}\right\rangle=\left(\frac{k T}{2 \pi m}\right)^{1 / 2} \sum_{i k i^{\prime} k^{\prime}} \frac{\left(\mathrm{i}_{2}+1\right)\left(k_{2}+1\right)}{Z_{\text {int }}^{2}} \int\left(\Delta \mathcal{E}^{v i b r}\right)^{2} \gamma^{3} \exp \left(-\gamma^{2}-\frac{\varepsilon_{i}}{k T}-\frac{\varepsilon_{k}}{k T}\right) \sigma_{i k}^{i^{\prime} k^{\prime}} \mathrm{d}^{2} \Omega \mathrm{d} \gamma
$$

При расчете этого интеграла использовались следующие допущения: сечения колебательных энергетических переходов вычисляются как сечения упругих столкновений, умноженные на вероятности соответствующих переходов; упругие столкновения описываются с помощью модели твердых сфер. При таких предположениях мы получаем аналитическую формулу, зависящую от вероятностей перехода. Вероятности переходов $\mathrm{VT}_{2}$ и $\mathrm{VV}_{m-k}$ вычисляются с использованием SSH-теории [26].

Так как число колебательных переходов в молекуле $\mathrm{CO}_{2}$ составляет около $10^{6}$ (см. [15]), то прямое суммирование требует значительных вычислительных усилий. Для предварительных оценок времени релаксации учитываются все переходы $\mathrm{VT}_{2}$ и несколько VV переходов между низшими колебательными состояниями. Такое приближение обеспечивает быструю и эффективную качественную оценку времен колебательной релаксации. Отметим, что поскольку дефект резонанса колебательной энергии при VV переходах мал, их 
влияние на характерные времена и объемную вязкость оказывается малым; расчеты показали, что разница не превышает $0.1 \%$. Это подтвердило обоснованность сформулированного выше приближенного подхода.

На рис. 1 время колебательной $\mathrm{VT}_{2}$ релаксации сравнивается со временем релаксации, измеренным экспериментально [25]. Времена даны как функции от $T^{-1 / 3}$. Для сравнения с результатами работы [25] из уравнения (13) исключались VV переходы, таким образом, мы получили $\tau_{V T_{2}}$. Время колебательной $\mathrm{VT}_{2}$ релаксации по формулам (13), (14) считалось с учетом различного числа колебательных переходов и учитываемых колебательных уровней. Сравнение показывает, что при низких температурах (до 500 K) для корректного теоретического расчета достаточно учитывать только нижние состояния; однако при $T>1000 \mathrm{~K}$ разница существенно увеличивается, и необходим учет всех возбужденных состояний. При низких температурах рассчитанные времена релаксации близки к измеренным. Интересно отметить, что если учитывать не все возможные колебательные состояния, то теоретическая зависимость времени $\mathrm{VT}_{2}$ релаксации от температуры становится немонотонной. Причем чем меньше уровней учитывается, тем раньше достигается минимальное значение $\tau_{V T_{2}}$ и тем больше оно растет.

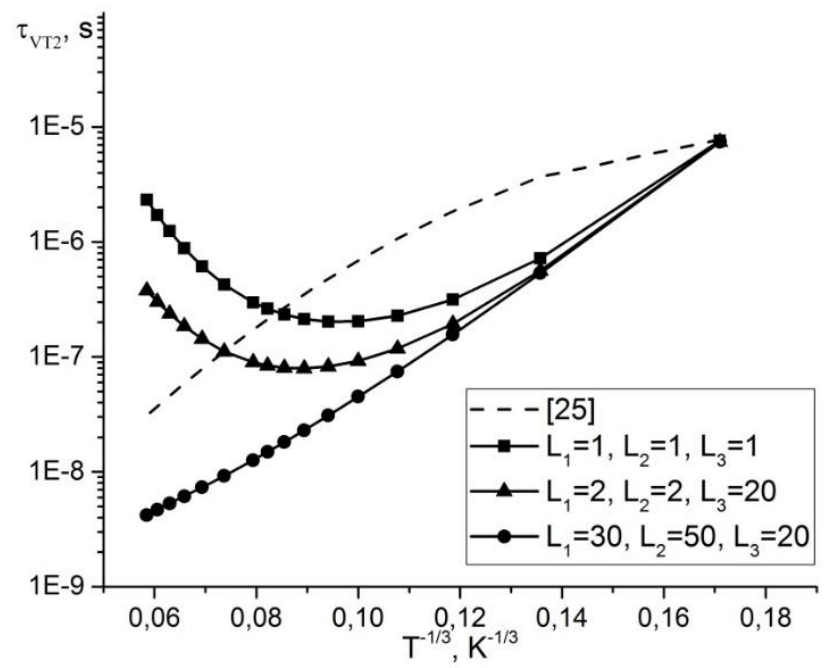

Рис. 1. Характерные времена $\mathrm{VT}_{2}$ обменов. Сравнение результатов, полученных методами кинетической теории с экспериментальными данными [25]

На рис. 2 время вращательной и колебательной релаксации сравнивается с временем релаксации внутренней энергии, вычисленным с использованием уравнения (9). Можно заметить, что зависимость $\tau_{\text {int }}$ от температуры немонотонна. При низких температурах температурная зависимость $\tau_{\text {int }}$ аналогична температурной зависимости $\tau_{r o t}$, тогда как $\tau_{v i b r}$ определяет поведение $\tau_{\text {int }}$ при высоких температурах. 


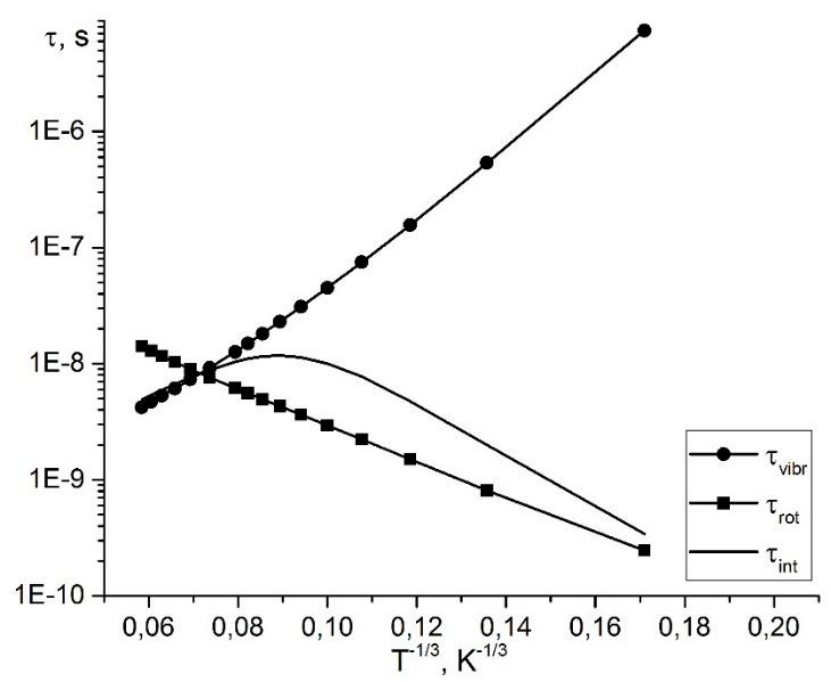

Рис. 2. Характерные времена в $\mathrm{CO}_{2}$. Сравнение $\tau_{\text {rot }}, \tau_{v i b r}$ и $\tau_{\text {int }}$, полученных методами кинетической теории

Теперь рассмотрим коэффициент объемной вязкости. Чтобы сравнить наши результаты с результатами Крамера [17], мы вычислили $\zeta$, а также два независимых вклада $\zeta^{\text {rot }}$ и $\zeta^{v i b r}$ по формулам (12) и их сумму (11). На рис. 3 мы сравниваем отношение $\left(\zeta^{r o t}+\zeta^{v i b r}\right) / \eta$, полученное с использованием различных данных для времени колебательной релаксации. При $T>1000$ К получаем очень хорошее совпадение с результатами Крамера. При низких температурах существует расхождение, вызванное использованием различных моделей для времен релаксации; результаты Крамера основаны на экспериментах Симпсона [27], тогда как мы используем как теоретическую формулу (13), так и эксперименты из [25].

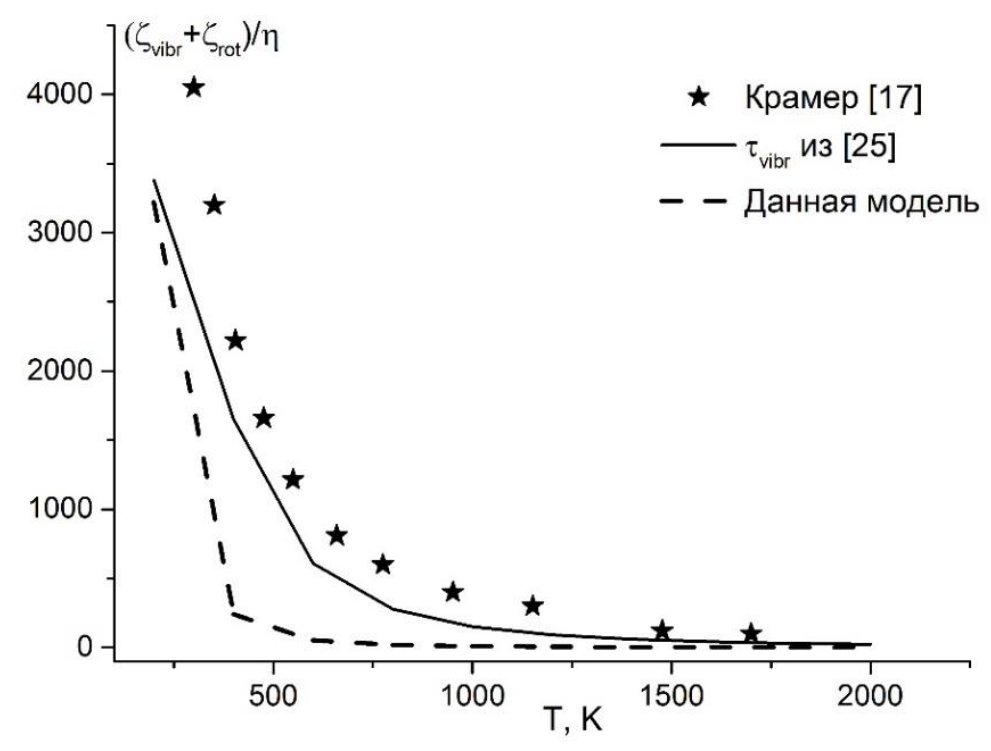

Рис. 3. Зависимость $\left(\zeta^{r o t}+\zeta^{v i b r}\right) / \eta$ от температуры в $\mathrm{CO}_{2}$

Следует отметить, что в формулировке Крамера основной вклад в объемную вязкость при низких температурах дают колебательные степени свободы. Действительно, $\zeta^{v i b r}$ на несколько порядков больше, чем $\zeta^{r o t}$; поэтому их сумма в основном не зависит от вращательных степеней свободы. Однако если вычислить $\zeta$ с использованием точной формулировки кинетической теории (10), то основную роль играют вращательные степени свободы. Это неудивительно, так как при низких температурах колебательные степени свободы можно рассматривать как замороженные по сравнению с вращательными. Следует отметить, 
что в случае $\tau_{r o t} \ll \tau_{v i b r}$ колебательная релаксация не может быть описана в рамках однотемпературной модели, предполагающей слабое отклонение от равновесия; медленные релаксационные процессы должны быть описаны с использованием релаксационных уравнений, полученных в сильнонеравновесных условиях.

На рис. 4 представлено отношение $\zeta / \eta$, вычисленное с использованием моделей кинетической теории (10) при учете различного количества колебательных переходов. Кроме того, приведено отношение коэффициентов, вычисленное при условии замороженных колебательных степеней свободы с учетом только вращательного возбуждения. В противоположность результатам [17], отношение $\zeta / \eta$ не превосходит 5 при $T=2000$ К. Видим, что при учете большего количества колебательных переходов рост отношения коэффициента объемной вязкости к сдвиговой замедляется, а при учете всех колебательных переходов $\zeta / \eta$ ведет себя немонотонно, достигая максимальной величины 3 при $T \sim 1000$ К. Колебательная релаксация заметно влияет на объемную вязкость в рассмотренном диапазоне температур, однако ее вклад имеет тот же порядок, что вклад вращательной релаксации. При высоких и низких температурах вклад колебательных степеней свободы в объемную вязкость уменьшается, и основную роль играют вращательные степени свободы.

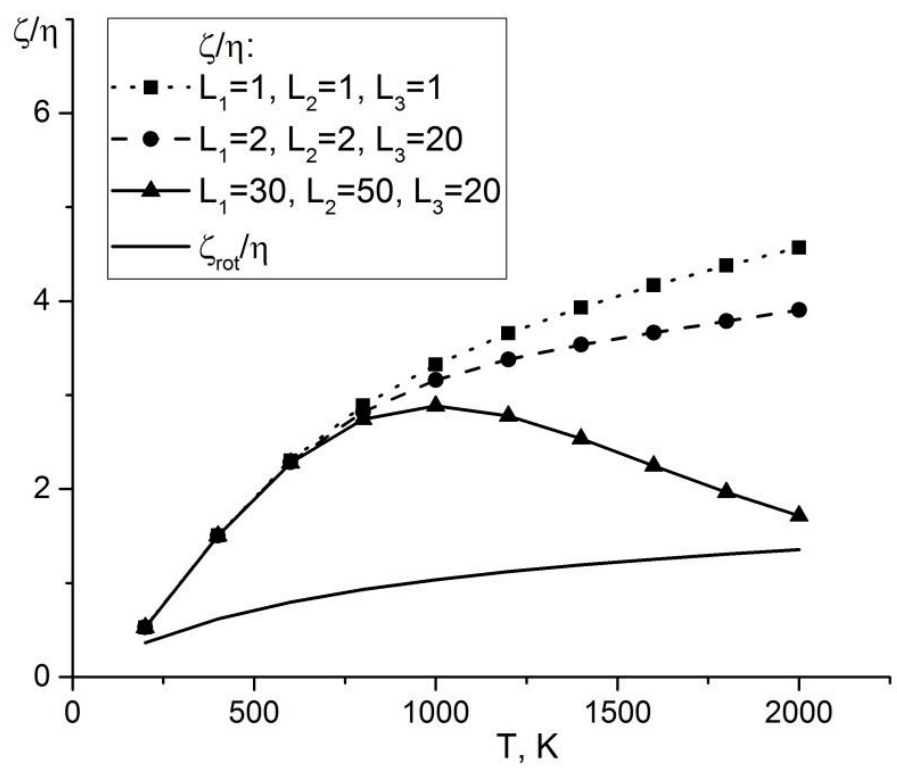

Рис. 4. Зависимость $\zeta / \eta$ от температуры в $\mathrm{CO}_{2}$

\section{4. Заключение}

Обсуждаются две модели для коэффициента объемной вязкости в углекислом газе: одна основана на методах точной кинетической теории, другая представляет коэффициент объемной вязкости в виде суммы независимых вкладов вращательных и колебательных степеней свободы. Последняя модель предсказывает, что при низких температурах отношение коэффициента объемной вязкости к сдвиговой в $\mathrm{CO}_{2}$ может достигать нескольких тысяч. Показано, что применение последовательного теоретического подхода не позволяет разделить объемную вязкость на вращательные и колебательные вклады. Максимальное отношение $\zeta / \eta$, полученное при использовании точной модели, составляет около 3 при $T=1000 \mathrm{~K}$; вклад колебательных степеней свободы (практически замороженных в низкотемпературных условиях) оказывается ниже по сравнению с вращательными.

Другим важным результатом этой работы является общий алгоритм, разработанный для расчета времени колебательной релаксации с учетом возбуждения всех колебательных мод $\mathrm{CO}_{2}$ и множества каналов колебательной релаксации. Данный алгоритм может приме- 
няться в условиях, когда достоверные экспериментальные данные по времени релаксации отсутствуют в литературе.

\section{Благодарности и ссылки на гранты}

Работа выполнена при поддержке Российского Фонда Фундаментальных Исследований грант номер 18-31-00110.

\section{Литература}

1. G. Prangsma, A. Alberga, and J. Beenakker, Physica 64, 278-288 (1973).

2. P. Hermans, L. Hermans, and J. Beenakker, Physica A122, 173-211 (1983).

3. X. Pan, M. N. Shneider, and R. B. Miles, Phys. Rev. A 69, p. 033814 (2004).

4. Z. Gu and W. Ubachs, Optics Letters 38, 1110-1112 (2013).

5. A. Ern and V. Giovangigli, Eur. J. Mech., B/Fluids 14, 653-669 (1995).

6. V. Galkin and S. Rusakov, Journ. Applied Mathematics and Mechanics 69, 943-954 (2005).

7. G. Billet, V. Giovangigli, and G. de Gassowski, Combust. Theory Modell. 12, p. 221 (2008).

8. E. Kustova, "On the role of bulk viscosity and relaxation pressure in non-equilibrium flows," in Rarefied Gas Dynamics: 26th International Symposium, AIP Conference Proceedings, Vol. 1084, edited by T. Abe (American Institute of Physics, Melville, NY, 2009), pp. 807-812.

9. Нагнибеда Е.А., Кустова Е.В. Кинетическая теория процессов переноса и релаксации в потоках неравновесных реагирующих газов. СПб.: Изд-во СПбГУ, 2003, 270 с.

10. D. Bruno and V. Giovangigli, Phys. Fluids 23, p. 093104 (2011).

11. V. Istomin, E. Kustova, and M. Mekhonoshina, "Validity of Eucken formula and Stokes viscosity relation in high-temperature electronically excited gases," in Rarefied Gas Dynamics, AIP Conference Proceedings, Vol. 1628, edited by J. Fan (American Institute of Physics, Melville, NY, 2014), pp. 1229-1236.

12. E. Kustova, M. Mekhonoshina, and G. Oblapenko, Chem. Phys. Lett. 686, 161-166 (2017).

13. G. M. Kremer, O. Kunova, E. Kustova, and G. Oblapenko, Physica A 490, 92-113 (2018).

14. E. Kustova and E. Nagnibeda, "State-to-state theory of vibrational kinetics and dissociation in threeatomic gases," in Rarefied Gas Dynamics, AIP Conference Proceedings, Vol. 585, edited by T. Bartel and M. Gallis(American Institute of Physics, Melville, NY, 2001), pp. 620-627.

15. I. Armenise and E. Kustova, Chem. Phys. 415, 269-281 (2013).

16. I. Armenise and E. Kustova, J. Phys. Chem. A122, 5107-120 (2018).

17. M. S. Cramer, Phys. Fluids 24, p. 066102 (2012).

18. S. Kosuge and K. Aoki, Physical Review Fluids 3, p. 023401 (2018).

19. Алексеев И.В., Кустова Е.В., Вестник Санкт-Петербургского университета. Математика, Механика, Астрономия, 4, 642-653 (2017).

20. I. Alekseev, A. Kosareva, E. Kustova, and E. Nagnibeda, "Various continuum approaches for studying shockwave structure in carbon dioxide," in Eight Polyakhov's Reading, AIP Conference Proceedings, Vol. 1959, edited by E. Kustova and et al (American Institute of Physics, Melville, NY, 2018) p. 060001.

21. G. Herzberg, Infrared and Raman Spectra of Polyatomic Molecules. D.Van Nostrand Company, Inc., NewYork, 1951.

22. D. Bruno, M. Capitelli, C. Catalfamo, R. Celiberto, G. Colonna, P. Diomede, D. Giordano, C. Gorse, A. Laricchiuta, S. Longo, D. Pagano, and F. Pirani, "Transport properties of high-temperature Mars-atmosphere components," ESA STR 256 (ESA, Noordwijk: ESA Publications Division, 2008).

23. E. Mason and L. Monchick, J. Chem. Phys. 36, 1622-1632 (1962).

24. J. Parker, Phys. Fluids 2, p. 449 (1959). 
25. Ачасов О. В., Рагозин Д. С., "Константы колебательного энергообмена в лазерно-активных средах СО2-ГДЛ с добавками О2, Н2, Н2О, СО”: Препринт № 16. Минск, Белоруссия: ИТМО, 1986

26. R. Schwartz, Z. Slawsky, and K. Herzfeld, J. Chem. Phys. 20, p. 1591 (1952).

27. C. J. S. M. Simpson, K. B. Bridgman, and T. R. D. Chandler, J. Chem. Phys. 49, 509-513 (1968).

Статья поступила в редакцию 30 ноября 2018 г. 